\title{
Resultados de un programa de tratamiento de rehabilitación domiciliaria en pacientes hemipléjicos por enfermedad cerebrovascular
}

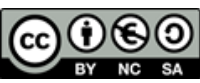

Results of a home rehabilitation treatment program in hemiplegic patients due to cerebrovascular disease

Maura Guerra Sánchez. ${ }^{1}$, Jayne Peñate Brito. ${ }^{2}$, Mónica González Guerra. ${ }^{3}$ \& Dayamí Cepero Silvera. ${ }^{4}$

Abstract.

Introduction: Cerebrovascular diseases are a global health problem, they are the first cause of disability in adults and the second cause of dementia. In Cuba, cerebrovascular accidents are the third leading cause of death for all ages. Cerebrovascular disease is the main cause of the appearance of the hemiplegic syndrome. Rehabilitation must begin

\section{Resumen.}

Introducción: Las enfermedades cerebrovasculares son un problema de salud mundial, constituyen la primera causa de discapacidad en el adulto y la segunda causa de demencia. En Cuba, los accidentes cerebrovasculares constituyen la tercera causa de muerte para todas las edades. La enfermedad cerebrovascular es la causa principal de la aparición del

\footnotetext{
1 Hospital Comandante Faustino Pérez Hernández, Matanzas. Cuba, proffis.mtz@infomed.sld.cu, https://orcid.org/ 0000-0002-7537-2760

${ }^{2}$ Policlínico José Jacinto Milanés, Matanzas. Cuba, jayne.mtz@infomed.sld.cu, iD https://orcid.org/ 00000002-0872-1692

3 Clínica Estomatológica III Congreso del PCC, Matanzas, Cuba, monicagonzalezguerra96@gmail.com, (iD) https://orcid.org/ 0000-0001-9861-3267

${ }^{4}$ Hospital Comandante Faustino Pérez Hernández, Matanzas. Cuba, ahernandezceperosilvera@ gmail.com (iD) https://orcid.org/0000-0003-4408-0404
} 
early and in a coordinated way and must be maintained during the different phases of health care. After the acute phase, home rehabilitation represents an opportunity of improvement for patients with residual disability. Objective: To evaluate the neurological and functional recovery in hemiplegic patients with cerebrovascular disease after home-based rehabilitation. Methodology: An intervention study was carried out in patients with a diagnosis of hemiplegia who attended the "José Jacinto Milanés" Rehabilitation Service in the period from January 2018 to January 2019. After applying the inclusion and exclusion criteria, the sample was constituted by 26 patients with whom we conducted the study after establishing informed consent. A program of individualized therapeutic physical exercises was designed at each patient's home, for eight weeks, carried out by the physiotherapists and supported by the family. Results: The male sex and the ages of 60 to 69 years predominated. Arterial Hypertension was the most frequent risk factor. At the end of treatment, $84,61 \%$ of the patients had a mild neurological condition and 46, $15 \%$ had a mild hypertonia and were independent. Conclusions: With homebased rehabilitative treatment, improvement can be achieved regarding neurological status, spasticity, and dependence on activities of daily living in hemiplegic patients due to cerebrovascular disease.

Keywords: Cerebrovascular disease, hemiplegia, home-based rehabilitation síndrome hemipléjico. La rehabilitación ha de iniciarse de forma precoz $y$ coordinada y mantenerse durante las diferentes fases de la atención sanitaria. Tras la fase aguda, la rehabilitación domiciliaria representa una oportunidad de mejora para los pacientes que presentan una discapacidad residual. Objetivo: Evaluar la recuperación neurológica $\mathrm{y}$ funcional en pacientes hemipléjicos con una enfermedad cerebrovascular después de una rehabilitación de base domiciliaria. Metodología: Se realizó un estudio de intervención en pacientes con diagnóstico de hemiplejía que acudieron al Servicio de Rehabilitación "José Jacinto Milanés" en el período de enero de 2018 a enero de 2019. Después de aplicar los criterios de inclusión y exclusión la muestra quedó constituida por 26 pacientes con los que realizamos el estudio luego de establecer el consentimiento informado. Se diseñó un programa de ejercicios físicos terapéuticos individualizado en el hogar, durante ocho semanas realizado por los fisioterapeutas y apoyado por la familia. Resultados: Predominó el sexo masculino y las edades de 60 a 69 años. La Hipertensión Arterial fue el factor de riesgo más frecuente. Al finalizar el tratamiento el $84,61 \%$ de los pacientes presentaban una afección neurológica leve y el $46,15 \%$ una hipertonía leve y eran independientes. Conclusiones: Con el tratamiento rehabilitador de base domiciliaria se logra mejorar el estado neurológico, la espasticidad y la dependencia en las actividades de la vida diaria del paciente hemipléjico por enfermedad cerebrovascular. 


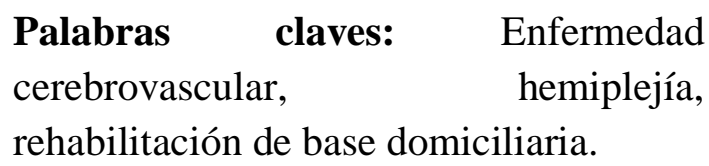

\section{Introducción.}

Las enfermedades cerebrovasculares son un problema de salud mundial, constituyen la primera causa de discapacidad en el adulto y la segunda causa de demencia. Según datos de la Organización Mundial de la Salud (OMS), 15 millones de personas sufren un ictus cada año; entre ellas, 5.5 millones mueren (el 10\% de todas las muertes producidas) y otros 5 millones quedan con alguna discapacidad permanente. (Ramírez Sterling M, Sentmanat Belisón A., 2018).

En Cuba, los accidentes cerebrovasculares constituyen la tercera causa de muerte para todas las edades; son la primera causa de urgencia médica y discapacidad entre las enfermedades neurológicas y más del $60 \%$ de los pacientes que sobreviven, requieren rehabilitación (Barroyeta Moreno D., 2018; Coll Costa, J.L, et al, 2017).

Entre los factores de riesgo están la hipertensión arterial, la hipercolesterolemia, la estenosis carotídea y la fibrilación auricular, ya que los resultados de ensayos clínicos han demostrado que el tratamiento de estas condiciones reduce su incidencia. El tabaquismo, el consumo excesivo de alcohol, la resistencia a la insulina y la diabetes mellitus también son factores de riesgo causales probables (Aviles Trujillo L. F., 2018). La enfermedad cerebrovascular es la causa principal de la aparición del síndrome hemipléjico y supone importantes gastos sanitarios, consumiendo gran cantidad de recursos económicos. (Vázquez Guimaraens M., 2017).

La hemiplejía consiste en una alteración de un lado del cuerpo y produce una afectación motora y/o alteración de la sensibilidad de ese hemicuerpo. Con frecuencia, además de la parálisis, quedan disminuidas otras funciones, como la visión, la capacidad auditiva, el habla e incluso aspectos cognitivos y conductuales. (Domínguez Ros Y., Elmacouti Bouhsain M., Villarreal Salcedo I., Cantín Cantín R., 2017; Paredes Ocaña S.E., 2020; Serrano Villota H.R., 2019).

Cuba realiza gran esfuerzo por mejorar la calidad de vida de su población; dentro de los programas de desarrollo que lleva a cabo la Salud Pública Cubana están los Servicios Integrales de Rehabilitación (SIR) con tecnología avanzada y estandarizada en la atención primaria de salud y extensión al domicilio, logrando hacer partícipe a la familia en la incorporación de pacientes discapacitados a la sociedad. (Coll Costa, J.L, et al, 2017).

La rehabilitación ha de iniciarse de forma precoz y coordinada y mantenerse durante las diferentes fases de la atención sanitaria. Tras la fase aguda, la rehabilitación domiciliaria representa una oportunidad de mejora para los pacientes que presentan una discapacidad residual tras el ictus. El hecho de realizar la evaluación en el propio entorno del paciente 
permitirá obtener una visión mucho más global y, sobre todo, más real de la discapacidad que presenta y, en especial, de cómo repercute en su vida cotidiana tanto física como socialmente. En muchos casos, el objetivo de la rehabilitación estará relacionado con la recuperación funcional y, en otros, con el mantenimiento de las capacidades residuales, la adaptación del domicilio o la educación sanitaria; no obstante, en todos los casos habrá un objetivo común, que será la mejora del bienestar y de la calidad de vida de los enfermos y sus familias (Vázquez Guimaraens M., 2017).

Teniendo en cuenta la dificultad que presentan muchos pacientes para trasladarse a los servicios de rehabilitación y por la necesidad del apoyo familiar para su recuperación, se realizó un estudio con el objetivo de evaluar la recuperación neurológica y funcional en pacientes hemipléjicos con una enfermedad cerebrovascular después de una rehabilitación de base domiciliaria.

\section{Metodologia.}

Se realizó un estudio de intervención, donde el universo de trabajo estuvo constituido por 38 pacientes con el diagnóstico de hemiplejía causada por una enfermedad cerebrovascular, que acudieron al Servicio Integral de Rehabilitación "José Jacinto Milanés“" desde enero 2018 a enero de 2019.

Después de aplicar los criterios de inclusión y exclusión la muestra quedó constituida por 26 pacientes con los que realizamos el estudio luego de establecer el consentimiento informado. Las Variables a estudiar fueron la edad, el sexo, factores de riesgo como: hipertensión arterial, hábito de fumar, diabetes mellitus, enfermedades cardiovasculares y el alcoholismo, las escalas de evaluación neurológica National Institute of Health,( NIH) y la escala de espasticidad de Ashworth e índice de Barthel. Estas escalas fueron aplicadas antes y después del tratamiento.

Se diseñó un programa de ejercicios físicos terapéuticos individualizado en el hogar, durante ocho semanas realizado por los fisioterapeutas y apoyado por la familia. En la primera y segunda semana se realizó el tratamiento diario en horario de la mañana y el familiar lo repetía en la tarde, luego las restantes seis semanas se visitó al paciente 3 veces en semana por el fisioterapeuta y los demás días lo ejecutaba el familiar entrenado. En la última semana se realizó la evaluación final por el fisiatra.

Los principios del tratamiento que se tuvo en cuenta fueron: cuidados postulares, respiración abdomino-diafragmática, movilizaciones pasivas de los miembros afectos o movilizaciones activas asistidas según examen físico, estimulación propioceptiva y sensorial del hemicuerpo afecto, conseguir el equilibrio sentado, adaptación progresiva a la verticalidad, ejercicios activos de aquellos grupos musculares con movimientos voluntarios, patrones de inhibición de la espasticidad, reeducación de la marcha con asistencia del familiar, terapia ocupacional para mejorar la coordinación y destreza manual con tareas del hogar como cambiar objetos de un lado para el otro, procurar la independencia en actividades de la vida diaria básica (vestido, aseo, comida.), psicoterapia de apoyo al paciente y al familiar. 


\section{Resultados.}

En investigaciones consultadas (López Egea J. 2015-2016; Sacre González M.R., Velázquez Valdés M.A., Castillo Ordaz M., Pastrana Trujillo L., Trujillo Morejón N., 2017) coincide con los autores que el comportamiento de los ACV se presenta con mayor frecuencia en pacientes del sexo masculino, mientras que en un estudio realizado en pacientes del Hospital General Docente Ambato los resultados muestran la mayor frecuencia en el sexo femenino (Áviles Trujillo L.F 2018), en este último las edades más frecuentes son los mayores de 60 años de edad.

En la tabla 1 aparecen reflejadas las edades entre 60 y 69 años como las más frecuentes de la muestra estudiada con un total de 15 pacientes para un $57.69 \%$, y el sexo masculino el de mayor porciento con 69,3.

Tabla 1. Distribución de pacientes hemipléjicos con rehabilitación domiciliaria según la edad y el sexo. SIR José Jacinto Milanés. Matanzas.

\begin{tabular}{llllllll}
\hline \multicolumn{1}{c}{ Sexo } & Femenino & \multicolumn{3}{c}{ Masculino } & \multicolumn{3}{c}{ Total } \\
\hline $\mathbf{6 0}$ a 69 Años & 5 & $19,3 \%$ & 10 & $38.4 \%$ & $\mathbf{1 5}$ & $57,69 \%$ \\
$\mathbf{7 0}$ a 79 Años & 3 & 11,55 & 5 & $19,3 \%$ & 8 & $30,79 \%$ \\
$\mathbf{8 0}$ y más Años & 0 & $0 \%$ & 3 & $11,5 \%$ & 3 & $11,5 \%$ \\
TOTAL & 8 & $30.79 \%$ & $\mathbf{1 8}$ & $69,3 \%$ & $\mathbf{2 6}$ & $\mathbf{1 0 0} \%$
\end{tabular}

\section{Fuente: Elaboración propia}

La Tabla 2 presenta los factores de riesgos encontrados. El 100\% tenían hipertensión arterial lo cual es el principal factor de riesgo para el ictus trombótico y el hemorrágico, su prevalencia aumenta con la edad y el riesgo de ictus aumenta proporcionalmente con las cifras de tensión arterial por lo que es el factor más importante en la prevención de las enfermedades cerebrovasculares. El hábito de fumar se muestra como otro factor de riesgo de gran importancia para estas enfermedades. Estudios precedentes como los de (López L., Ramos V., Katz E., Pérez V., Camarot T., 2017; Áviles Trujillo L.F 2018) identificaron como factor de riesgo principal en la aparición de la enfermedad, la hipertensión arterial, siendo el identificado en el presente estudio.

Tabla 2. Prevalencia de los factores de riesgo en los pacientes estudiados. SIR José Jacinto Milanés. Matanzas.

\begin{tabular}{lll}
\hline Factores de riesgo & Número & Porciento \\
\hline HTA & $\mathbf{2 6}$ & $100 \%$ \\
Hábito de fumar & 16 & $61,53 \%$ \\
\hline
\end{tabular}


Diabetes mellitus

Enfermedades cardiovasculares

Alcohol

12

12

10
$46,15 \%$

$46,15 \%$

$38,46 \%$

Fuente: Elaboración propia

La mejoría de los parámetros neurológicos evaluados en los pacientes se obtuvo por los procederes de rehabilitación que se realizaron en el domicilio tanto por los fisioterapeutas como por la familia, que en la mayoría de los casos tuvo una activa participación en el proceso, apropiándose de los conocimientos prácticos para la realización de los diferentes patrones kinésicos. Los pacientes consideraban muy positivo el poder rehabilitarse en su propio entorno domiciliario, se sentían muy atendidos por sus familiares y consideraban que las dificultades para el traslado al servicio de rehabilitación dejaba de constituir un problema.

Se considera que la evolución neurológica satisfactoria de los pacientes hemipléjicos se puede lograr desde el propio domicilio con un programa bien estructurado y con el apoyo de la familia. (Montagut Martínez F. et al. 2015) comparan la rehabilitación domiciliaria con la del hospital de día en pacientes con ACV y obtienen los mismos resultados funcionales, encontrando en el primer caso un menor coste hospitalario pero un aumento en la demanda de servicios sociales.

El estado neurológico según escala de NIH que se evalúa antes y después del tratamiento es reflejado en la tabla 3 . Al inicio el 46,15\% se encontraba con una afección neurológica moderada y al finalizar, los pacientes presentaron una afección neurológica leve en un $84,61 \%$, lo que evidenció la adecuada evolución de los pacientes al tratamiento impuesto.

Tabla 3. Evaluación del estado neurológico según escala de NIH antes y después del tratamiento rehabilitador. SIR José Jacinto Milanés. Matanzas

\begin{tabular}{|c|c|c|c|c|}
\hline \multirow[b]{2}{*}{ Puntuación } & \multicolumn{2}{|l|}{ Antes } & \multicolumn{2}{|l|}{ Después } \\
\hline & número & porciento & número & Porciento \\
\hline $\begin{array}{l}\text { Afectación } \quad \text { neurológica } \\
\text { ligera }(\leq 5)\end{array}$ & 7 & $26,92 \%$ & 22 & $84,61 \%$ \\
\hline $\begin{array}{l}\text { Afectación neurológica } \\
\text { moderada (5 a 15) }\end{array}$ & 12 & $46,15 \%$ & 4 & $15,38 \%$ \\
\hline $\begin{array}{l}\text { Afectación neurológica } \\
\text { grave (15 a 24) }\end{array}$ & 7 & $26,92 \%$ & 0 & $0 \%$ \\
\hline $\begin{array}{l}\text { Afectación neurológica } \\
\text { muy grave }(\geq 25)\end{array}$ & 0 & $0 \%$ & 0 & $0 \%$ \\
\hline total & 26 & $100 \%$ & 26 & $100 \%$ \\
\hline
\end{tabular}

Fuente: Elaboración propia 
La mejoría del tono muscular se atribuye a la apropiación del familiar que apoyaba en la rehabilitación del paciente de las técnicas de tratamiento rehabilitador explicadas por los fisioterapeutas, específicamente los patrones de inhibición de espasticidad, los adecuados tratamientos posturales y el uso de las ayudas ortésicas. Se considera que en el tratamiento de la hipertonía no es imprescindible el uso de agentes físicos terapéuticos cuando se aplican correctamente las técnicas kinésicas independientemente del lugar en que se realicen.

(Montagut Martínez F. et al. 2015) publicaron los buenos resultados y el alto grado de satisfacción de pacientes con ACV agudo y moderada espasticidad que seguían un programa de rehabilitación domiciliaria de 3 meses de duración, con una reducción del $52 \%$ de la estancia hospitalaria, comparándolo con programas de rehabilitación en el hospital.

La Tabla 4 refleja cómo después del tratamiento rehabilitador, se obtienen resultados satisfactorios en la disminución de los grados de espasticidad. Antes de iniciar el tratamiento ningún paciente presentaba un tono muscular normal, todos se encontraban en los grados 1,2, 3, 4 estando el mayor número de casos con hipertonía moderada (61,53\%), una vez culminado el tratamiento disminuye a un 19,23\%, incrementándose la mejoría del tono muscular con puntuación 1 y 0 en un 34,61\% y 46,15\%.

Tabla 4. Evaluación de la espasticidad según escala de Ashworth antes y después del tratamiento rehabilitador. SIR José Jacinto Milanés. Matanzas.

\begin{tabular}{lllll}
\hline \multirow{2}{*}{ Puntuación } & \multicolumn{2}{c}{ Antes } & & \\
& \multicolumn{2}{l}{ Después } \\
\cline { 2 - 5 } & número & porciento & número & Porciento \\
\hline $\mathbf{0}$ (tono muscular normal) & 0 & $0 \%$ & 9 & $\mathbf{3 4 , 6 1 \%}$ \\
$\mathbf{1}$ (hipertonía leve) & 2 & $7,69 \%$ & 12 & $\mathbf{4 6 , 1 5 \%}$ \\
$\mathbf{2}$ (hipertonía moderada) & 16 & $61,53 \%$ & 5 & $19,23 \%$ \\
$\mathbf{3}$ (hipertonía intensa) & 4 & $15,38 \%$ & 0 & $0 \%$ \\
$\mathbf{4}$ (hipertonía extrema) & 4 & $15,38 \%$ & 0 & $0 \%$ \\
total & & & & $100 \%$ \\
\hline
\end{tabular}

Fuente: Elaboración propia

Para obtener los grados de dependencia se utilizó el Índice de Barthel, siendo utilizado también en un estudio para determinar la estancia hospitalaria (Palomino Peña C. A., 2018). Se atribuye que las capacidades funcionales de los pacientes mostraron evidente mejoría por la adecuada recuperación neurológica y por la posibilidad del entrenamiento 
de las actividades de la vida diaria en el propio entorno donde se desarrolla el paciente, realizando así las adecuaciones necesarias para su mejor realización.

En los grados de dependencia en las actividades de la vida diaria, Tabla 5, se observó que al inicio del tratamiento todos los pacientes presentaban cierto grado de dependencia principalmente entre leve y moderada $38,46 \%$ y $46,15 \%$ respectivamente. Al finalizar el tratamiento encontramos que el $46,15 \%$ de los pacientes ya eran independientes para las Actividades de la vida diaria y un $38,46 \%$ eran dependientes leves.

Tabla 5. Evaluación de las actividades de la vida diaria según índice de Barthel antes y después del tratamiento rehabilitador. SIR José Jacinto Milanés. Matanzas.

\begin{tabular}{lllll}
\hline Puntuación & antes & \multicolumn{3}{l}{ Después } \\
& \multicolumn{1}{l}{ no } & $\%$ & no & $\%$ \\
\hline independiente (100) & 0 & $0 \%$ & 12 & $46,15 \%$ \\
dependiente leve ( $\geq$ 60) & 12 & $46,15 \%$ & 10 & $38,46 \%$ \\
dependiente moderado (40a59) & 7 & $26,92 \%$ & 4 & $15,38 \%$ \\
dependiente grave (20 a 39) & 5 & $19,23 \%$ & 0 & $0 \%$ \\
dependiente total (< 20) & 2 & $7,69 \%$ & 0 & $0 \%$ \\
total & 26 & $100 \%$ & 26 & $100 \%$
\end{tabular}

Fuente: Elaboración

Los autores estiman que los resultados obtenidos en la investigación demuestran que la rehabilitación domiciliaria más que una opción, es un modelo de tratamiento, que con una adecuada planificación, entrenamiento, participación familiar y control, arroja grandes beneficios para la recuperación funcional de los pacientes hemipléjicos con una enfermedad cerebrovascular y una mejor calidad de vida para la familia.

\section{Conclusiones.}

- Predominó el sexo masculino y el rango de edad de 60 a 69 años.

- La HTA constituyó el principal factor de riesgo.

- Con el tratamiento rehabilitador de base domiciliaria se logra mejorar el estado neurológico, la espasticidad y la dependencia en las actividades de la vida diaria del paciente hemipléjico por enfermedad cerebrovascular.

\section{Referencias bibliográficas.}

Avilés, L. (2018). Prevalencia de las secuelas de la enfermedad cerebrovascular en adultos mayores atentidos en el Hospital General Docente Ambato. Proyecto de 
Investigación previo a la obtencion de título de Médico Cirujano. Facultad de Ciencias Médicas, Ambato, Ecuador.

Barroyeta, D. (2018). Tratamiento neurocognitivo y rehabilitación en paciente hemipléjico. Universidad Inca Garcilaso De La Vega. Facultad de Tecnología Médica, Lima, Perú .

Coll, J. (2017). Programa de ejercicios físicos para la rehabilitación del paciente hemipléjico. La Habana, Cuba: Editorial Deportes.

Domínguez, Y., Elmacouti, M., Villarreal, I., \& Cantín, R. (2017). Plan de intervención desde terapia ocupacional en un paciente afecto de hemiplejía derecha: tratamiento rehabilitador centrado en funcionalidad de extremidad superior. Revista TOG (A Coruña), 14(26).

López, J. (2020). Rehabilitación Neuropsicológica de Inicio Tardío: Valoración de la Funcionalidad en Pacientes con Daño Cerebral Adquirido. Universidad Miguel Hernández, España.

López, L., Ramos, V., Katz, E., Pérez, V., \& Camarot, T. (2017). Resultados Funcionales y Complicaciones en pacientes asistidos en UDA-ACV Rehabilitación Instituto de Neurología- Hospital de Clínicas . Revista Uruguay Medicina Interna (2), 3342.

Montagut, F., Flotats, G., \& Lucas, E. (2015). Rehabilitación domiciliaria. Principios, indicaciones y programas terapéuticos ( $2^{\mathrm{a}}$ edición ed.). España: Elsevier Masson.

Palomino, C. (2018). Escalas de Barthel y medida de independencia funcional para determinar la duración de estancia hospitalaria y plan terapéutico rehabilitador accidente cerebrovascular, Hospital II Clínica Geriátrica San Isidro Labrador. Universidad de San Martín de Porres, Lima, Perú .

Paredes, S. (2020). Bobath en la intervención fisioterapéutica de la hemiplejía. Universidad Nacional de Chimborazo. Facultad de Ciencias de la Salud, Riobamba, Ecuador.

Ramírez, M. d., \& Sentmanat, A. (2018). Batería de ejercicios físicos para la rehabilitación de los miembros superiores en pacientes con hemiplejía asistidos en la casa, en Cali, Colombia. Revista de la Facultad de Cultura Física de la Universidad de Granma, 15(51).

Sacre, M. d., Velázquez, M., Castillo, M., Pastrana, L., \& Trujillo, N. (2017). Caracterización de los cuidadores de adultos mayores hemipléjicos a causa de enfermedad cerebrovascular . Revista MEDISAN, 21(8).

Serrano, H. (2019). Fisiopatogenia de la Hemiplejía en adultos. Un análisis fisioterapéutico. Universidad Nacional de Chimborazo. Facultad de Ciencias de la Salud, Riobamba, Ecuador. 
Vázquez, M. (2017). Factores relacionados a una mayor recuperación funcional tras sufrir un accidente cerebrovascular. Universidad de La Coruña, Ciencias Biomédicas, Medicina y Fisioterapia, España.

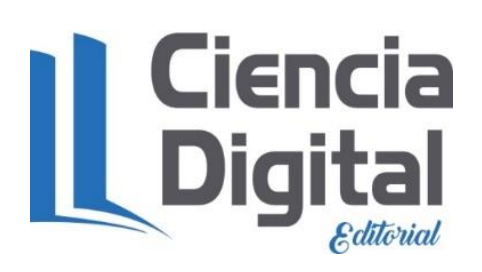




\section{PARA CITAR EL ARTÍCULO INDEXADO.}

Guerra Sánchez, M., Peñate Brito, J., González Guerra, M., \& Cepero Silvera, D. (2021). Resultados de un programa de tratamiento de rehabilitación domiciliaria en pacientes hemipléjicos por enfermedad cerebrovascular . Anatomía Digital, 4(3), 76-86. https://doi.org/10.33262/anatomiadigital.v4i3.1757

\section{Ciencia \\ LDigital}

El artículo que se publica es de exclusiva responsabilidad de los autores y no necesariamente reflejan el pensamiento de la Revista Anatomía Digital.

El artículo queda en propiedad de la revista y, por tanto, su publicación parcial y/o total en otro medio tiene que ser autorizado por el director de la Revista Anatomía Digital.
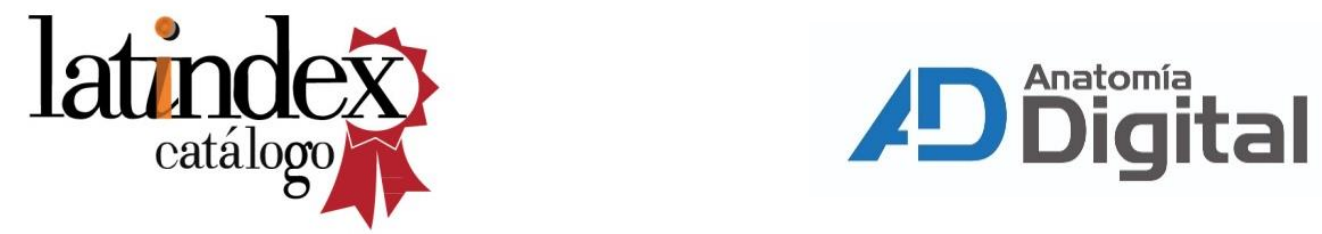